\title{
Misbehaving macrophages in the pathogenesis of psoriasis
}

\author{
Rachael A. Clark and Thomas S. Kupper
}

Harvard Skin Disease Research Center and Department of Dermatology, Brigham and Women's Hospital, Boston, Massachusetts, USA.

\begin{abstract}
Psoriasis is a chronic inflammatory skin disease unique to humans. In this issue of the JCI, 2 studies of very different mouse models of psoriasis both report that macrophages play a key role in inducing psoriasis-like skin disease. Psoriasis is clearly a polygenic, inherited disease of uncontrolled cutaneous inflammation. The debate that currently rages in the field is whether psoriasis is a disease of autoreactive $T$ cells or whether it reflects an intrinsic defect within the skin - or both. However, these questions have proven difficult to dissect using molecular genetic tools. In the current studies, the authors have used 2 different animal models to address the role of macrophages in disease pathogenesis: Wang et al. use a mouse model in which inflammation is $T$ cell dependent, whereas the model used by Stratis et al. is $T$ cell independent (see the related articles beginning on pages 2105 and 2094, respectively). Strikingly, both groups report an important contribution by macrophages, implying that macrophages can contribute to both epithelial-based and $\mathrm{T}$ cell-mediated pathways of inflammation.
\end{abstract}

\section{Psoriatic skin: a riot of disorder}

Psoriatic skin is characterized by dense infiltrates of $\mathrm{T}$ cells and cells of the innate immune system, including neutrophils, dendritic cells, macrophages, and NKT cells. The epithelial cells of the skin are hyperproliferative and fail to undergo normal differentiation, leading to a marked thickening of the epidermis. There are dramatic increases in the number and size of blood vessels situated just below the epidermis, and abscesses composed of neutrophils form within the epidermis. The clinical result is red, thickened, and flaking skin. Psoriasis is a chronic, lifelong aggravation for many patients because skin lesions can be treated in the short term but they tend to recur when treatment is discontinued. In addition to its clinical impact, psoriasis is a fascinating and accessible model in which to study inflammation within the skin.

\section{What is to blame in psoriasis - T cells or the skin?}

Changes in the epidermis are the most striking feature of psoriasis, which led to the earliest hypotheses that psoriasis is a

Nonstandard abbreviations used: IKK2, inhibitor of NF-KB kinase 2; MCP-1, monocyte chemotactic protein 1.

Conflict of interest: The authors have declared that no conflict of interest exists.

Citation for this article: J. Clin. Invest. 116:2084-2087 (2006). doi:10.1172/JCI29441. disorder of the epidermis. However, findings that $\mathrm{T}$ cell immunosuppressants such as cyclosporine and $\mathrm{T}$ cell-depleting agents such as denileukin diftitox (Ontak) lead to disease remission forced a re-evaluation of this hypothesis and a refocusing on the role of immune cells (1). Based on many observations, including the fact that psoriasis can be induced in SCID mouse xenograft models by the injection of $\mathrm{T}$ cells from human skin lesions, it is largely accepted that psoriasis is a $\mathrm{T}$ cell-dependent disease $(2,3)$. More recently, an elegant model of psoriasis was developed in which nonlesional, unaffected skin from patients with psoriasis transplanted onto immunodeficient mice was found to spontaneously develop true human psoriatic lesions. The population of $\mathrm{T}$ cells resident within the nonlesional skin from psoriatic patients was triggered, presumably by danger signals released by the trauma of grafting, to proliferate in a TNF- $\alpha-$ dependent manner, leading to the development of psoriatic skin lesions (4). Together, these studies suggest that there is a pool of autoreactive $T$ cells present within the normal skin of psoriatic patients that can proliferate when stimulated by APCs within the dermis. However, autoantigens for psoriasis have not been conclusively identified, so definitive assignment of psoriasis as an autoimmune disorder awaits further study.

Psoriasis is unique to humans, but it has been modeled with varying degrees of suc- cess using genetically manipulated mice. Many psoriasis mouse models require the participation of $\mathrm{T}$ cells for development of skin inflammation (4-8), but other models appear able to recapitulate the histologic changes of psoriasis without the requirement for $\mathrm{T}$ cell participation (9-11). One such $\mathrm{T}$ cell-independent model, used by Stratis et al. in this issue of the $J C I$, is generated by deletion of inhibitor of NF-KB kinase 2 (IKK2) specifically within the epidermis (12). Mice with this defect develop inflammatory lesions within the skin that have many similarities to the histologic changes seen in psoriasis (10). Proponents of these models argue that the intrinsic defect in psoriasis may well lie within the skin and, more specifically, that psoriatic skin cells may overproduce inflammatory mediators that can engender and perpetuate inflammatory reactions independent of the action of autoreactive $\mathrm{T}$ cells (13).

\section{Macrophages contribute to psoriasis} in $\mathbf{2}$ mouse models

Infiltration of macrophages, especially around the epidermal/dermal interface, is a well-established feature of human psoriasis, but the contribution of these cells to psoriatic inflammation was previously unknown (14-19). In this issue, Wang et al. (20) and Stratis et al. (12) address for the first time to our knowledge the importance of macrophages in mouse models of psoriasis. Using 2 different mouse models, one $T$ cell dependent and one skin dependent, both groups found that macrophages are present in large numbers in psoriasis-like skin lesions and that these cells play a key role in the pathogenesis of psoriasiform skin inflammation.

In their report, Wang et al. (20) used mice that express decreased levels of CD18. These CD18 hypomorphic mice spontaneously develop chronically inflamed skin lesions that histologically resemble human psoriasis (7). Skin inflammation in this model is polygenic, $\mathrm{T}$ cell dependent, and responsive to topical steroids, all features common to human psoriasis. Wang et al. demonstrate 


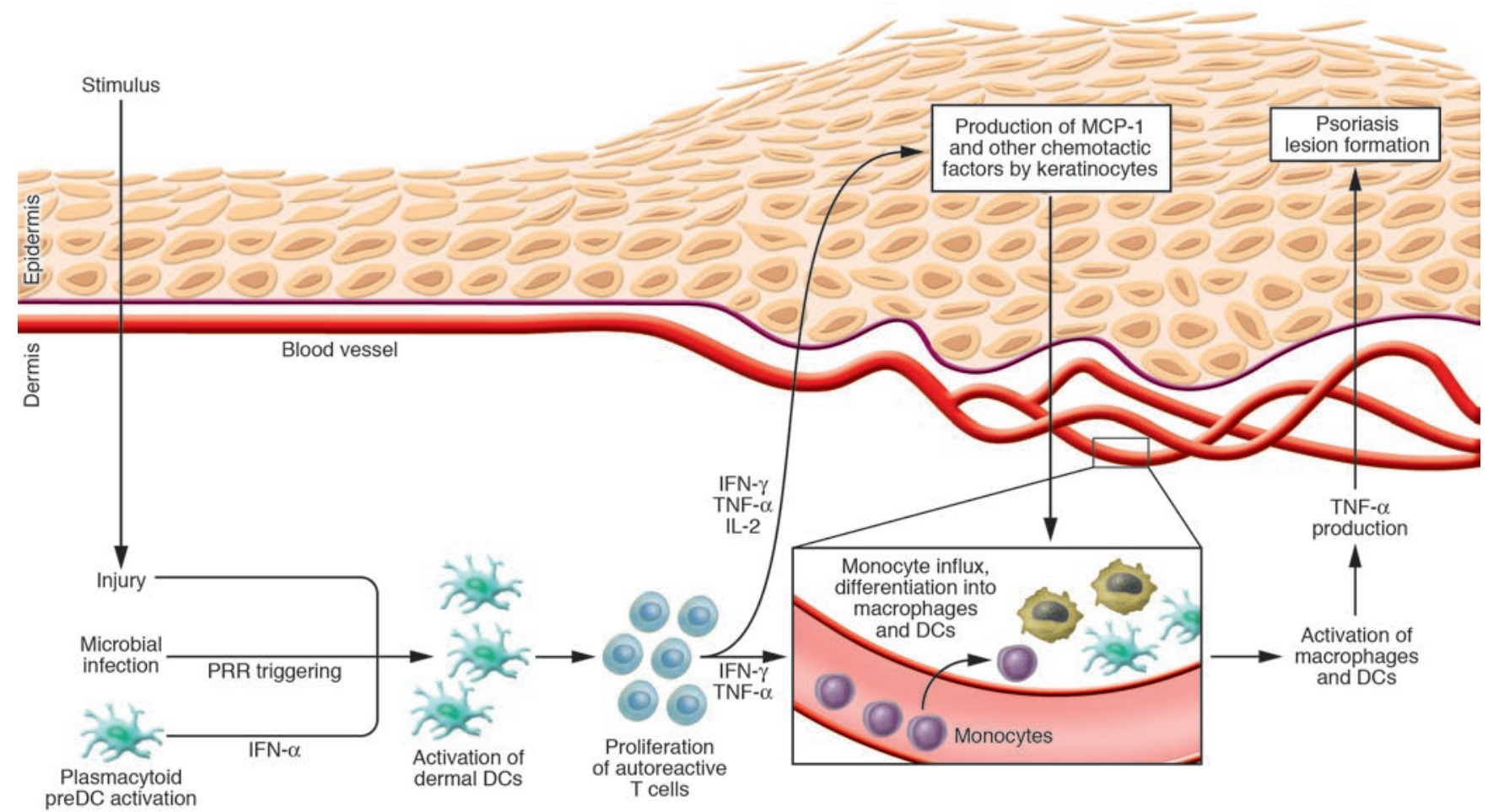

\section{Figure 1}

An emerging model of psoriasis pathogenesis in humans. Many insults can lead to the activation of dermal dendritic cells, a key initiating step in the development of psoriasis in predisposed individuals. Activated dendritic cells induce the proliferation of autoreactive T cells within the dermis, inducing production of IFN- $\gamma$ and TNF- $\alpha$, which in turn induces the production of MCP-1 and other chemotactic cytokines by epidermal cells. These chemotactic agents induce influx of monocytes from the blood, which undergo differentiation into macrophages and myeloid dendritic cells. The studies of Stratis et al. (12) and Wang et al. (20) reported in this issue of the $\mathrm{JCl}$ suggest that these dermal macrophages, once activated by $\mathrm{T}$ cell or dendritic cell cytokines, then produce large amounts of TNF- $\alpha$, leading to the skin changes observed in psoriasis. PRR, pathogen-recognition receptor.

that skin lesions contain large numbers of macrophages and that these cells are a significant source of TNF- $\alpha$ within the skin. Blockade of TNF- $\alpha$ signaling by etanercept, a drug effective in the treatment of human psoriasis, improved psoriasiform skin inflammation, decreased the numbers of macrophages in skin lesions, and decreased production of TNF- $\alpha$.

To more specifically evaluate the contribution of macrophages, Wang et al. used clodronate liposomes to deplete macrophages from skin lesions (20). Clodronatetreated skin lesions lacked macrophages but had normal numbers of T cells, neutrophils, mast cells, and mature Langerhans cells. Treated skin showed significant improvements in inflammation and had vastly reduced levels of TNF- $\alpha$. The expression of monocyte chemoattractant protein 1 (MCP-1), a chemokine known to mediate cutaneous recruitment of monocytes and macrophages, was increased in lesional skin. However, although cutaneous injection of MCP-1 into nonlesional sites did induce macrophage migration into the skin, it did not induce psoriasis-like skin inflammation unless combined with injection of TNF- $\alpha$. Likewise, injection of TNF- $\alpha$ alone did not induce inflammation, suggesting that both migration of macrophages into the skin and their subsequent activation are required for the induction of skin disease. However, once skin-resident macrophages were activated, in this case by TNF- $\alpha$, they were capable of establishing and maintaining psoriasis-like skin inflammation. The authors conclude that macrophage recruitment and activation are key features in the development of psoriasiform inflammation. Interestingly, depletion of GR-1-positive neutrophils by neutralizing antibodies did not inhibit inflammation, suggesting that these cells, although present in large numbers in psoriasis, do not contribute to skin inflammation.

In the study by Stratis et al. (12), the authors used a mouse model of psoriasis in which epidermal-specific deletion of IKK2 leads to a chronic inflammatory skin disorder with histologic similarities to human psoriasis (10). However, unlike xenogenic models of psoriasis that use human skin $(2,4)$, inflammation in this model is not $\mathrm{T}$ cell dependent. Stratis et al. also demonstrate that macrophages are present in large numbers in skin lesions, that treatment with TNF antagonists improves skin inflammation, and that clodronate treatment depletes macrophages and leads to an improvement in skin disease. To address the role of neutrophils, these animals were crossed to CD18-deficient mice and still developed skin disease, again confirming that granulocytes such as neutrophils do not play a pathogenic role in the inflammatory process. Lastly, this group found no reduction in skin inflammation in IFN- $\gamma$ deficient mice, arguing against a key role for IFN- $\gamma$ in this model of psoriasis.

It is striking that 2 disparate psoriatic models in mice $(12,20)$, one $\mathrm{T}$ cell dependent and one $\mathrm{T}$ cell independent, provide such concordant results with respect to the importance of activated macrophages in the initiation and maintenance of psoriasiform skin inflammation. Both groups found large numbers of macrophages 
within skin lesions and found that depletion of these cells, along with other phagocytic cells in the skin, leads to a significant improvement in skin inflammation. In both reports, the macrophages present in skin lesions expressed both classical and alternative activation makers, in agreement with findings in human psoriasis $(16,19)$. Both groups found no contribution of granulocytes to the underlying pathogenesis of skin inflammation, a significant observation given that dense infiltrates of neutrophils are a feature in both human and mouse models of psoriasis. Wang et al. (20) make a significant contribution by identifying macrophages as crucial sources of TNF- $\alpha$, along with previously identified sources that include $T$ cells, mast cells, and endothelial cells $(21,22)$. TNF- $\alpha$ is a critical cytokine in the pathogenesis of psoriasis, as shown by the remarkable effectiveness of etanercept, a TNF- $\alpha$-neutralizing agent, in the treatment of human psoriasis $(23,24)$.

In short, these 2 studies have produced remarkably concordant results regarding the importance of macrophages in psoriasis. However, a disorder that looks histologically like psoriasis in mice may not necessarily reflect the underlying etiology or complexity of the human disease. For this reason, it will be important to replicate these findings in human or xenogenic systems.

\section{Many roads lead to psoriasis}

The reports by Wang et al. (20) and Stratis et al. (12) both suggest that once macrophages are activated to produce large amounts of TNF- $\alpha$, they induce all the skin changes characteristic of psoriasiform dermatitis, at least in the mouse models under study. The next logical question is, what induces the accumulation and activation of macrophages in psoriatic skin? In the model used by Wang et al., T cells are required for macrophage recruitment, as previously shown by the fact that depletion of $\mathrm{CD}^{+} \mathrm{T}$ cells from these mice induced complete resolution of skin disease (25). Xenogenic models of psoriasis have shown that human psoriatic lesion development is T cell dependent, and indeed, migration of $\mathrm{CD}^{+} \mathrm{T}$ cells precedes the accumulation of macrophages in human psoriatic lesions $(2,4,26)$. These $\mathrm{T}$ cells must themselves become activated, likely through interaction with activated dermal dendritic cells. Figure 1 illustrates an emerging model of psoriasis pathogenesis in which activation of dermal dendritic cells has central importance. In this model, autoreactive $\mathrm{T}$ cells and dendritic cells express- ing autoantigens populate the normal skin of patients with psoriasis. These cells do not cause inflammation in their resting, nonactivated state, but their close proximity within the skin is an accident waiting to happen. The key event is likely to be the origination of a "danger signal" within the skin, which is then translated into dermal dendritic cell activation. Many stimuli can lead to the activation of dermal dendritic cells, including injury, infection with organisms that trigger dendritic cell pathogen-recognition receptors, and activation of plasmacytoid predendritic cells (27-29). Once dermal dendritic cells are activated, they spark the activation of autoreactive $T$ cells, leading to their proliferation within the dermis $(4,30)$. Activated dendritic cells and autoreactive $\mathrm{T}$ cells then secrete inflammatory cytokines that induce the production of MCP-1 and other chemotactic factors by keratinocytes, leading to an influx of macrophages and dendritic cells. The TNF- $\alpha$ produced by these and other cells then drives the development of the skin changes we observe in psoriasis. The claim that multiple insults can lead to psoriasis in predisposed individuals is consistent with several clinical observations. First, psoriasis patients can develop new lesions in areas of previously unaffected skin after injury, an observation known as the Koebner phenomenon. Second, a particular form of psoriasis is clearly associated with streptococcal pharyngitis. It is worth restating that all known forms of human psoriasis are absolutely dependent upon the actions of T cells. Scientific studies demonstrating $T$ cell dependence are bolstered by the clinical observation that almost all patients with psoriasis experience remission of their skin disease when treated with $\mathrm{T}$ cell-specific agents such as cyclosporine.

In the mouse psoriasis model used by Stratis et al. (12), macrophage recruitment and activation were mediated by signals provided by keratinocytes with impaired NF-кB signaling, not by the activation of autoreactive $T$ cells. Although there is no evidence supporting $\mathrm{T}$ cell-independent psoriasis in humans, it is true that a very small number of patients do not respond to treatment with T cell-immunosuppressive medications. It is possible that abnormal sensitivity to inflammatory stimuli within the skin may lead to psoriasis in this small subset of patients.

In conclusion, the recruitment and activation of macrophages in psoriatic skin appears to be a key pathogenic event in the development and maintenance of psoriatic skin disease. The underlying events that lead to macrophage recruitment and activation remain to be fully characterized and may result from either autoreactive $\mathrm{T}$ cells or aberrant epidermal signals. Further clarification of what instigates the misbehavior of these cells will lead to treatments that can strike at the true source of this chronic inflammatory skin disease.

\section{Acknowledgments}

T.S. Kupper is supported by NIH grants P50 CA-93683-04, P30 AR-42689-11, RO1 AI41707-09, R37 AI-25082-17, 1U19 AI5733001, and HHSN266200400030C. R.A. Clark is supported by NIH grant 1K08AI06089001A1, a Translational Research Program Award from the Leukemia and Lymphoma Foundation, and a Pilot Grant from the Harvard Skin Disease Research Center.

Address correspondence to: Thomas S. Kupper, Harvard Skin Disease Research Center and Department of Dermatology, Brigham and Women's Hospital, Harvard Institutes of Medicine 670, 77 Avenue Louis Pasteur, Boston, Massachusetts 02115, USA. Phone: (617) 525-5550; Fax: (617) 525-5571; E-mail: tkupper@partners.org.

1. Olsen, E., et al. 2001. Pivotal phase III trial of two dose levels of denileukin diftitox for the treatment of cutaneous T-cell lymphoma. J. Clin. Oncol. 19:376-388.

2. Wrone-Smith, T., and Nickoloff, B.J. 1996. Dermal injection of immunocytes induces psoriasis. J. Clin. Invest. 98:1878-1887.

3. Prinz, J.C. 2003. The role of T cells in psoriasis. J. Eur. Acad. Dermatol. Venereol. 17:257-270.

4. Boyman, O., et al. 2004. Spontaneous development of psoriasis in a new animal model shows an essential role for resident $T$ cells and tumor necrosis factor-alpha. J. Exp. Med. 199:731-736.

5. Sano, S., et al. 2005. Stat3 links activated keratinocytes and immunocytes required for development of psoriasis in a novel transgenic mouse model. Nat. Med. 11:43-49.

6. Schon, M.P., Detmar, M., and Parker, C.M. 1997. Murine psoriasis-like disorder induced by naive CD4+ T cells. Nat. Med. 3:183-188.

7. Bullard, D.C., et al. 1996. A polygenic mouse model of psoriasiform skin disease in CD18-deficient mice. Proc. Natl. Acad. Sci. U. S. A. 93:2116-2121.

8. Nickoloff, B.J., and Wrone-Smith, T. 1999. Injection of pre-psoriatic skin with CD4+ T cells induces psoriasis. Am. J. Pathol. 155:145-158.

9. Schon, M., Denzer, D., Kubitza, R.C., Ruzicka, T., and Schon, M.P. 2000. Critical role of neutrophils for the generation of psoriasiform skin lesions in flaky skin mice. J. Invest. Dermatol. 114:976-983.

10. Pasparakis, M., et al. 2002. TNF-mediated inflammatory skin disease in mice with epidermis-specific deletion of IKK2. Nature. 417:861-866.

11. Zenz, R., et al. 2005. Psoriasis-like skin disease and arthritis caused by inducible epidermal deletion of Jun proteins. Nature. 437:369-375.

12. Stratis, A., et al. 2006. Pathogenic role for skin macrophages in a mouse model of keratinocyte- 
induced psoriasis-like skin inflammation. J. Clin. Invest. 116:2094-2104. doi:10.1172/JCI27179.

13. Djalilian, A.R., et al. 2006. Connexin 26 regulates epidermal barrier and wound remodeling and promotes psoriasiform response. J. Clin. Invest. 116:1243-1253. doi:10.1172/JCI27186.

14. Gillitzer, R., et al. 1993. MCP-1 mRNA expression in basal keratinocytes of psoriatic lesions. J. Invest. Dermatol. 101:127-131.

15. van den Oord, J.J., and de Wolf-Peeters, C. 1994. Epithelium-lining macrophages in psoriasis. Br. J. Dermatol. 130:589-594.

16. Djemadji-Oudjiel, N., Goerdt, S., Kodelja, V., Schmuth, M., and Orfanos, C.E. 1996. Immunohistochemical identification of type II alternatively activated dendritic macrophages (RM $3 / 1+3$, MS-1+/-, 25F9-) in psoriatic dermis. Arch. Dermatol. Res. 288:757-764.

17. Vestergaard, C., et al. 2004. Expression of CCR2 on monocytes and macrophages in chronically inflamed skin in atopic dermatitis and psoriasis. Acta Derm. Venereol. 84:353-358.

18. Boehncke, W.H., Wortmann, S., Kaufmann, R. Mielke, V., and Sterry, W. 1995. A subset of mac- rophages located along the basement membrane ("lining cells") is a characteristic histopathological feature of psoriasis. Am. J. Dermatopathol. 17:139-144.

19. Nickoloff, B.J. 2000. Characterization of lymphocyte-dependent angiogenesis using a SCID mouse: human skin model of psoriasis. J. Investig. Dermatol. Symp. Proc. 5:67-73

20. Wang, H., et al. 2006. Activated macrophages are essential in a murine model for $\mathrm{T}$ cell-mediated chronic psoriasiform skin inflammation. J. Clin. Invest. 116:2105-2114. doi:10.1172/JCI27180.

21. Schlaak, J.F., et al. 1994. T cells involved in psoriasis vulgaris belong to the Th1 subset. J. Invest. Dermatol. 102:145-149.

22. Ackermann, L., and Harvima, I.T. 1998. Mast cells of psoriatic and atopic dermatitis skin are positive for TNF-alpha and their degranulation is associated with expression of ICAM-1 in the epidermis. Arch. Dermatol. Res. 290:353-359.

23. Gottlieb, A.B., et al. 2003. A randomized trial of etanercept as monotherapy for psoriasis. Arch. Dermatol. 139:1627-1632; discussion 1632.

24. Leonardi, C.L., et al. 2003. Etanercept as mono- therapy in patients with psoriasis. N. Engl. J. Med. 349:2014-2022.

25. Kess, D., et al. 2003. CD4+ T cell-associated pathophysiology critically depends on CD18 gene dose effects in a murine model of psoriasis. J. Immunol. 171:5697-5706.

26. Paukkonen, K., Naukkarinen, A., and Horsmanheimo, M. 1992. The development of manifest psoriatic lesions is linked with the invasion of CD8 $+\mathrm{T}$ cells and CD11c + macrophages into the epidermis. Arch. Dermatol. Res. 284:375-379.

27. Banchereau, J., and Steinman, R.M. 1998. Dendritic cells and the control of immunity. Nature. 392:245-252.

28. Janeway, C.A., Jr., and Medzhitov, R. 2002. Innate immune recognition. Annu. Rev. Immunol. 20:197-216.

29. Nestle, F.O., et al. 2005. Plasmacytoid predendritic cells initiate psoriasis through interferon-alpha production. J. Exp. Med. 202:135-143.

30. Nestle, F.O., Turka, L.A., and Nickoloff, B.J. 1994. Characterization of dermal dendritic cells in psoriasis. Autostimulation of T lymphocytes and induction of Th1 type cytokines. J. Clin. Invest. 94:202-209.

\title{
Tuning the oviduct to the anandamide tone
}

\author{
Herbert Schuel
}

Division of Anatomy and Cell Biology, Department of Pathology and Anatomical Sciences, School of Medicine and Biomedical Sciences, University at Buffalo, State University of New York, Buffalo, New York, USA.

\begin{abstract}
Anandamide ( $N$-arachidonoylethanolamide) is a lipid signal molecule that was the first endogenous agonist for cannabinoid receptors to be discovered. Cannabinoid receptor type 1 (CB1) is widely distributed in neurons and nonneuronal cells in brain and peripheral organs including sperm, eggs, and preimplantation embryos. A study by Wang and colleagues in this issue of the JCI demonstrates that a critical balance between anandamide synthesis by $N$-acylphosphatidylethanolamine-selective phospholipase D (NAPE-PLD) and its degradation by fatty acid amide hydrolase (FAAH) in mouse embryos and oviducts creates locally an appropriate "anandamide tone" required for normal embryo development, oviductal transport, implantation, and pregnancy (see the related article beginning on page 2122). Adverse effects of elevated levels of anandamide on these processes resulting from FAAH inactivation are mimicked by administration of (-)- $\Delta^{9}$-tetrahydrocannabinol (THC; the major psychoactive constituent of marijuana), due to enhanced signaling via CB1. These findings show that exogenous THC can swamp endogenous anandamide signaling systems, thereby affecting multiple physiological processes.
\end{abstract}

Attempts to understand the mechanisms responsible for the psychoactive properties of tetrahydrocannabinol (THC) in marijuana led to the discovery of canna-

Nonstandard abbreviations used: 2-AG, 2-arachidonoylglycerol; CB1, cannabinoid receptor type 1; FAAH, fatty acid amide hydrolase; NAPE-PLD, $N$-acylphosphatidylethanolamine-selective phospholipase D; THC, $(-)-\Delta^{9}$-tetrahydrocannabinol.

Conflict of interest: The author has declared that no conflict of interest exists.

Citation for this article: J. Clin. Invest. 116:2087-2090 (2006). doi:10.1172/JCI29424. binoid receptors and their endogenous ligands, the endocannabinoids (1). Cannabinoid receptors are $G$ protein-coupled receptors. Two subtypes have been cloned and characterized to date. Cannabinoid receptor type 1 (CB1) was originally cloned from rat and human brain, while CB2 was originally cloned from human promyelocytic leukemia HL-60 cells. Anandamide and related fatty acid derivatives that activate these receptors are endocannabinoids. Anandamide is released from membrane phospholipid precursors by
$N$-acylphosphatidylethanolamine-selective phospholipase D (NAPE-PLD) when neurons and other cells are stimulated by neurotransmitters and hormones. Released anandamide is quickly eliminated by membrane-bound fatty acid amide hydrolase (FAAH), indicative of a role for this lipid in cell signaling.

Anandamide signaling via cannabinoid receptors is not restricted to the central nervous system. We now know that CB1, CB2, NAPE-PLD, and FAAH are widely distributed in nonneuronal somatic cells of peripheral organs including those of the reproductive system, as well as in germ cells of vertebrates and invertebrates (1-8). This may account for the effects of marijuana and THC on multiple aspects of reproductive physiology, including secretion of gonadotrophic hormones by the pituitary gland, secretion of sex steroids by gonads, sperm production, ovulation, mating behavior, sperm capacitation, fertilization, early embryonic development, implantation of blastocysts into the uterine endometrium, placental functions, fetal growth, number of pregnancies carried to term, lactation, suckling behavior of newborns, postnatal development, and growth of malignant breast and prostate cells. 